EPJ Web of Conferences 19, 04001 (2012)

DOI: $10.1051 /$ epjconf/20121904001

(C) Owned by the authors, published by EDP Sciences, 2012

\title{
Chemical constraints on the formation of the Galactic thick disk
}

\author{
T. Bensby and S. Feltzing \\ Lund Observatory, Department of Astronomy and Theoretical Physics, Lund, Sweden
}

\begin{abstract}
We highlight some results from our detailed abundance analysis study of 703 kinematically selected $\mathrm{F}$ and $\mathrm{G}$ dwarf stars in the solar neighbourhood. The analysis is based on spectra of high-resolution ( $R=45000$ to 110000 ) and high signal-to-noise ( $S / N \approx 150$ to 300 ). The main findings include: (1) at a given metallicity, the thick disk abundance trends are more $\alpha$-enhanced than those of the thin disk; (2) the metal-rich limit of the thick disk reaches at least solar metallicities; (3) the metal-poor limit of the thin disk is around $[\mathrm{Fe} / \mathrm{H}] \approx-0.8 ;(4)$ the thick disk shows an age-metallicity gradient; (5) the thin disk does not show an age-metallicity gradient; (6) the most metal-rich thick disk stars at $[\mathrm{Fe} / \mathrm{H}] \approx 0$ are significantly older than the most metal-poor thin disk stars at $[\mathrm{Fe} / \mathrm{H}] \approx-0.7 ;(7)$ based on our elemental abundances we find that kinematical criteria produce thin and thick disk stellar samples that are biased in the sense that stars from the low-velocity tail of the thick disk are classified as thin disk stars, and stars from the high-velocity tail of the thin disk are classified as thick disk stars; (8) age criteria appears to produce thin and thick disk stellar samples with less contamination.
\end{abstract}

\section{INTRODUCTION}

Thick disks as unique entities in galaxies were discovered in the late 1970s when it was found that the vertical light profiles of a few edge-on galaxies could not be fitted by single exponentials [1]. Similarly, the Galactic thick disk as a unique stellar population was detected in the early 1980:s when star count data towards the Galactic South Pole could not be fitted with just one power law but needed two: one with a scale-height of $300 \mathrm{pc}$ and one with a scale-height of $1350 \mathrm{pc}$ [2]. The former was associated with the already known thin disk and the latter with the newly discovered thick disk. As there is no a priori reason that says that vertical star counts in galaxy disks must fit single power laws this finding was necessary but not sufficient to define the thick disk as a unique entity. Since then it has been shown that the Galactic thin and thick disks are also distinct disk populations in terms of kinematics as well as chemistry: the thick disk is a more slowly rotating stellar system than the thin disk, lagging the local standard of rest (LSR) by 40 to $50 \mathrm{~km} \mathrm{~s}^{-1}$ [3]; it is older than the thin disk [4, 5]; it has a lower average metallicity than the thin disk [6]; and, at a given metallicity, it is more $\alpha$-enhanced than the thin disk $[4,7-10]$. These differences point to separate origins and formation histories of the two disks. Also, it has recently been found that the Galactic bulge has a bimodal metallicity distribution [11, 12] and that the metal-poor part of the bulge is very similar to the thick disk (average metallicity, elemental abundance trends, and age distribution) $[11,13,14]$. This might suggest that the bulge and the thick disk are tightly connected.

From the Geneva-Copenhagen Survey (GCS) $[15,16]$, which contains kinematics, ages, and metallicities estimated from Strömgren uvby $\beta$ photometry for $\sim 14000$ nearby dwarf stars, it is evident that stars with orbital rotational velocities typical for the thick disk can be found at very high metallicities, even well above solar (see Fig. 1). The question is if these stars are true thick disk stars? Indeed, there has been studies that claim that the most metal-rich thick disk stars lies around

This is an Open Access article distributed under the terms of the Creative Commons Attribution-Noncommercial License 3.0, which permits unrestricted use, distribution, and reproduction in any noncommercial medium, provided the original work is properly cited. 


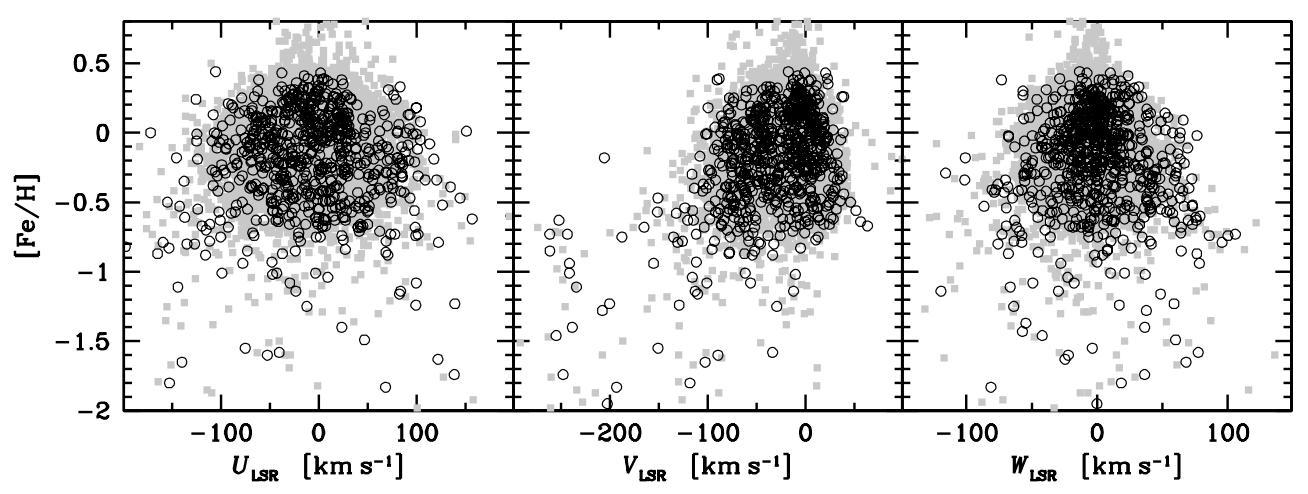

Figure 1. $[\mathrm{Fe} / \mathrm{H}]$ versus $U_{\mathrm{LSR}}, V_{\mathrm{LSR}}$, and $W_{\mathrm{LSR}}$ velocities for our stars (o). The grey dots in the background represent the $\sim 14000$ stars in the GCS catalogue using the new metallicities from [16].

$[\mathrm{Fe} / \mathrm{H}] \approx-0.3$ and that the metal-richer ones are likely to be thin disk stars due to their low $[\mathrm{a} / \mathrm{Fe}]$ ratios $[7,17,18]$. However, recent studies show that the thick disk can be traced to at least solar metallicities $[10,19]$.

To further investigate the chemical and kinematical properties of the Galactic disk, and the degree of the separation between the two disks, we have undertaken a large spectroscopic survey of $F$ and $\mathrm{G}$ dwarf stars in the Solar neighbourhood. The stellar sample consists of 703 stars and is the joint effort from several observing projects, each with its own specific goal, aiming at probing the different stellar populations in the Solar neighbourhood at their extremes. Important scientific questions addressed in our projects include: (i) How metal-rich can the thick disk be? (ii) How metal-poor can the thick disk be? (iii) How metal-poor can the thin disk be? (iv) How metal-rich can the halo be? (v) Where do the old and metal-rich stars come from? (vi) Where do kinematical substructures such as the Hercules stream and the Arcturus moving group come from? First results regarding points (iii) and (vi) can be found in [10] and [20]. Bringing these questions together will help us to constrain the formation history of the Milky Way, and will ultimately add pieces to the puzzle of galaxy formation.

\section{OBSERVATIONS AND ABUNDANCE ANALYSIS}

The stars were selected mainly from the GCS and a few, that were not available in the GCS, from the catalogue by Feltzing \& Holmberg [21]. Figure 1 shows [Fe/H] as a function of the $U_{\mathrm{LSR}}, V_{\mathrm{LSR}}$, and $W_{\text {LSR }}$ velocities. One aspect of our sample that sets it apart from, e.g., the studies by Reddy et al. [7, 22], is that we cover a wider range of orbital parameters. For example our sample includes stars on orbits with low eccentricity and low $[\mathrm{Fe} / \mathrm{H}]$ (chosen to study the metal-weak thin disc) as well as stars with super-solar $[\mathrm{Fe} / \mathrm{H}]$ and highly eccentric orbits and/or highly negative $V_{\mathrm{LSR}}$ velocities (chosen to study the metal-rich thick disc). Neither of these types of stars have been systematically included in previous studies. In fact in some studies they are lacking all together and the inclusion of them in our study enables us to explore a wider range of the parameter space. Figure 1 shows that we explore the full $[\mathrm{Fe} / \mathrm{H}]$-velocity plane of the GCS with high-resolution spectroscopy.

The methods to determine the stellar parameters and to calculate elemental abundances follow the methodology described in our previous papers that contain a subset of 102 stars of the current sample $[8,9]$. Further descriptions regarding the analysis, updated error estimation methods, and new age determination, will be included in an upcoming paper (Bensby, Feltzing \& Oey, in prep.). 

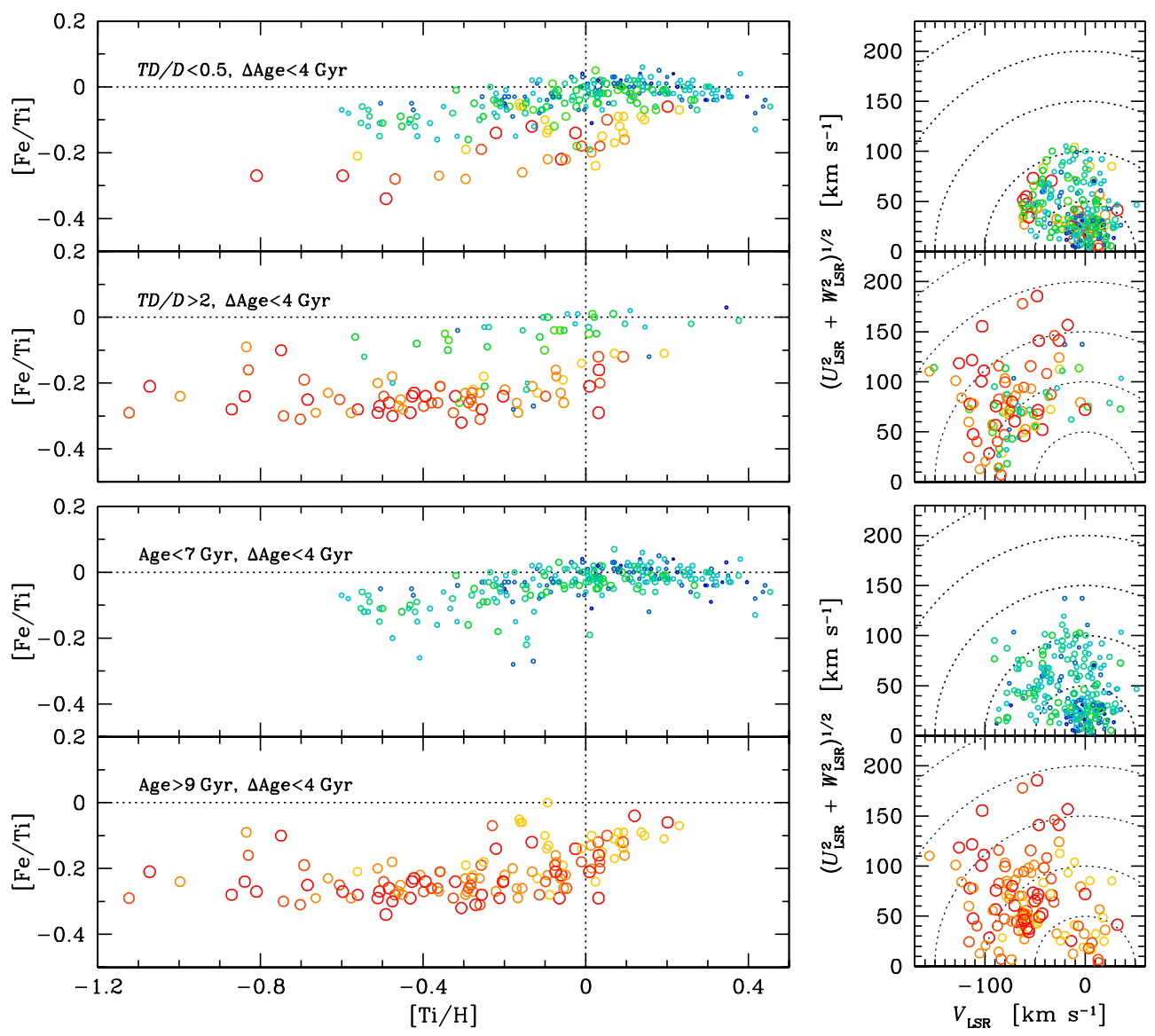

Figure 2. Upper left: $[\mathrm{Fe} / \mathrm{Ti}]-[\mathrm{Ti} / \mathrm{H}]$ abundance trends when using the kinematical criteria from $[8,9]$; lower left: when using age criteria. Right: Toomre diagrams for the samples on the left-hand side. Only stars with a difference between upper and lower age estimates less than 4 Gyr are shown. Stars have been colour- and size coded depending on their ages (blue and small circles represent young stars, red and big circles represent old stars).

\section{RESULTS AND DISCUSSION}

\subsection{Selection criteria}

Thick disk stellar candidates have usually been selected based on kinematical criteria [8, 9]. The reason to use kinematical criteria is because you want to investigate the chemical and age properties of the stellar populations, and if chemical and/or age criteria are invoked those investigations would be undermined. Using kinematical criteria, it has generally been found that stars with thick disk kinematics are more enhanced in the $\alpha$-elements than the stars with thin disk kinematics at a give metallicity $[7-9,17,22]$, and that they are older at all metallicities, even where the metallicities are over-lapping $[4,10,23]$.

In the upper left plot in Fig. 2 we show the $[\mathrm{Fe} / \mathrm{Ti}]-[\mathrm{Ti} / \mathrm{H}]$ abundance trends for two kinematically selected samples; one where the probabilities of being a thin disk star is at least twice that of being a thick disk star $(T D / D<0.5)$, and one where the probabilities of being a thick disk star is at least twice that of being a thin disk star $(T D / D>2)$. As can be seen the two samples show very different abundance trends. However, it is also clear that the main chemical signature for each kinematical sample appears to 

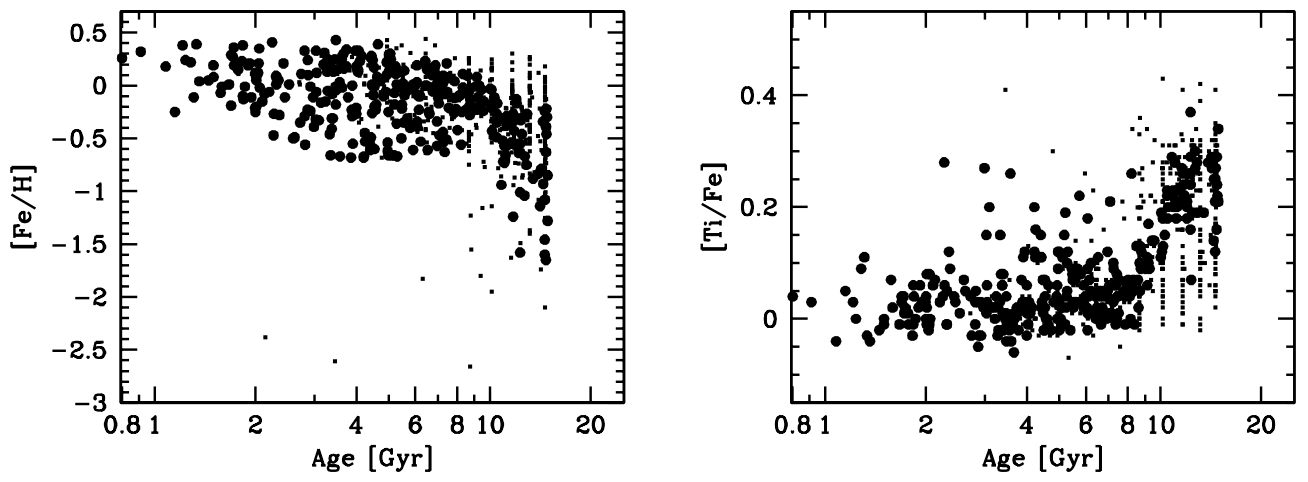

Figure 3. $[\mathrm{Fe} / \mathrm{H}]$ and $[\mathrm{Ti} / \mathrm{Fe}]$ versus age. Stars with well-determined ages (difference between upper and lower age estimate less than $3 \mathrm{Gyr}$ ) are marked by larger dots.

be present also in the other sample. We have therefore also in Fig. 2 coded each star by its estimated age. It is evident that the weaker signature in each kinematical sample has the same age structure as the main signature in the other sample, i.e. the $\alpha$-enhanced stars in the thin disk sample with $T D / D<0.5$ are older than the ones that are not as enhanced, and have the same ages as the $\alpha$-enhanced stars in the thick disk sample with $T D / D>2$. Vice versa, the stars with low $\alpha$-enhancements in the thick disk sample are younger than the ones with higher $\alpha$-enhancements, and have ages comparable with the bulk of stars in the thin disk sample. The Toomre diagrams on right-hand side of Fig. 2 show that the two samples are kinematically different, with only little overlap.

The $[\mathrm{Fe} / \mathrm{Ti}]-[\mathrm{Ti} / \mathrm{H}]$ abundance trends for two samples, one old sample with stars that have estimated ages greater than $9 \mathrm{Gyr}$, and one young sample with stars that have estimated age less than $7 \mathrm{Gyr}$, are shown in the bottom left plots of Fig. 2. Once again we see two very different chemical signatures, similar to the ones where the stars were separated based on their kinematics. However, the abundance trends are now much cleaner, with essentially no overlap between the two. Looking at the Toomre diagrams for these two samples (bottom right plots of Fig. 2) we see that there is a large kinematical overlap between the two. There are young stars with hot kinematics and old stars with cold kinematics.

It appears that kinematical selection criteria introduces a "kinematical confusion" between thin and thick disk stars. Due to the different metallicity distributions of the thin and thick disks, the problem is especially severe at high metallicities, where stars from the high-velocity tail of the thin disk are likely to be mis-classified as thick disk stars, and at low metallicities, where stars from the low-velocity tail of the thick disk are likely to be mis-classified as thin disk stars. Instead, stellar ages appear to be able to produce "cleaner" thin and thick disk samples. However, as stellar ages require knowledge of stellar parameters and metallicities, and are also only possible to determine for dwarf and subgiant stars, kinematics might be the only available discriminator. In those cases, one should be aware of the possible kinematical confusion between thin and thick disk stellar samples.

\subsection{Ages and metallicities}

Figure 3 show the age-metallicity plot for the stars with estimated ages. We highlight those stars that have well-determined ages, i.e., age difference between upper and lower estimates smaller than $3 \mathrm{Gyr}$. A few noticeable features are: (1) There are no young and metal-poor stars; (2) There are old and metal-rich stars; (3) The old and metal-rich stars all have ages with large error bars; (3) For stars with well-determined ages greater than $\approx 8 \mathrm{Gyr}$, there appears to exist an age-metallicity relation.

Also, from Fig. 2 we see that the most metal-rich stars with thick disk kinematics are significantly older than the most metal-poor stars with thin disk kinematics. Whether or not there is a real hiatus in 

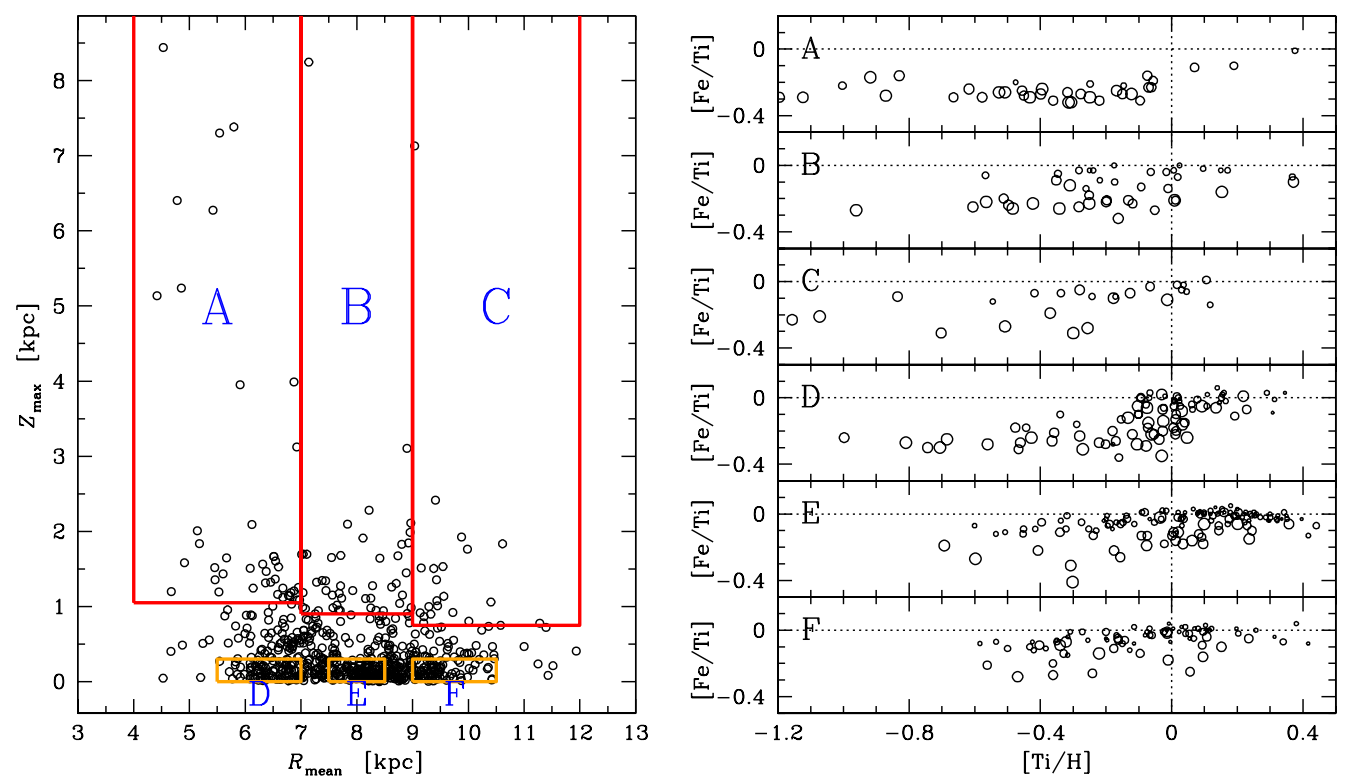

Figure 4. Left: $z_{\max }$ versus $R_{\text {mean }}$. Right: [Fe/Ti]-[Ti/H] for stars with orbital parameters as shown in boxes A-F in the plot on the left-hand side. The sizes of the circles have been scaled with the estimated ages of the stars.

the star formation history between the thin and thick disks is unclear, and requires further investigation with an un-biased sample. However, it is likely that the Milky Way, after star formation in the thick disk ceased (at $[\mathrm{Fe} / \mathrm{H}] \approx 0$ ), acquired gas of lower metallicity (merger?). Once star formation started again, it did so in the thin disk at lower metallicities $([\mathrm{Fe} / \mathrm{H}] \approx-0.7)$.

\subsection{Variation with galactocentric distance}

Figure 4 shows $z_{\max }$ as a function of $R_{\text {mean }}$ for the stellar sample. $R_{\text {mean }}$ can be regarded as an estimate of the distance from the Galactic centre where the stars were born [24]. Boxes A, B, and C contain stars on orbits that reach far from the Galactic plane, presumably born in the inner disk, at the solar distance, and in the outer disk, respectively, while Boxes D, E, and F contain stars on orbits close to the plane, presumably born in the inner disk, at the solar distance, and in the outer disk, respectively. The right-hand side plot of Fig. 4 shows the $[\mathrm{Fe} / \mathrm{Ti}]-[\mathrm{Ti} / \mathrm{H}]$ plots for the stars in these different boxes. The sizes of the markers have been scaled with the ages of the stars (older stars - bigger circles). First, we note that stars born at the solar distance (Boxes B and E) divide into the typical abundance trends we associate with the thin and thick disks. Box B contains more stars with thick disk properties (old and $\alpha$-enhanced) while Box E contains more stars with thin disk properties (young and no $\alpha$-enhancement). This is expected. If the relative properties of the inner and outer disk were similar to what is seen in the solar neighbourhood (Box E), the abundance trends of the stars in Boxes D and F should be similar to Box E. They are not. Instead Box D (inner disk) appears to contain only old stars with thick disk abundance ratios, and only very few young stars with thin disk abundance ratios. The opposite is true for Box F (outer disk) which mainly contains young stars with thin disk abundance ratios and only a very few old stars with thick disk abundance ratios. This means that the relative properties of the thin and thick disks changes when going from galactocentric distances $\left(R_{G}\right)$ of $6 \mathrm{kpc}$ to $8 \mathrm{kpc}$ to $10 \mathrm{kpc}$, in the sense that the thin disk dominates at large $R_{G}$ while the thick disk dominates at small $R_{G}$. If this is a real signature or just an effect of the various biases we have in our sample is difficult to say. We have been selecting stars on eccentric orbits in order to probe the thick disk, but no distinction has been 
made on if their orbits place them in the inner or outer disk. However, recent observations of distant $\mathrm{K}$ giants indicate that the abundance trends of the outer disk is dominated by stars with thin disk abundance ratios, and that this might be a consequence that the scale length of the thick disk is significantly shorter than that of the thin disk $[25,26]$. What we see in Boxes D and F could be a manifestation of that. If the thick disk scale length is shorter than the thin disk, we expect the thin disk to dominate more and more with galactocentric distance.

\subsection{Formation of the thick disk}

A number of formation scenarios for thick disks have been proposed, e.g.: heating by mergers [27, 28]; accretion of satellites [29]; early, clumpy rapid star formation [30]; and migration [31]. Although, it is likely that all of these processes to some extent act in the Milky Way, it is not clear which if any is the dominant mechanism.

Thick disk formation scenarios, together with the observational constraints imposed from our spectroscopic survey, will be discussed in detail in an upcoming paper (Feltzing, Bensby, \& Oey, in prep.). A full description of the sample of 703 stars, including abundances for $\mathrm{O}, \mathrm{Na}, \mathrm{Mg}, \mathrm{Al}, \mathrm{Si}, \mathrm{Ca}, \mathrm{Ti}$, $\mathrm{Fe}, \mathrm{Ni}, \mathrm{Zn}, \mathrm{Y}$ and $\mathrm{Ba}$, will be presented in another upcoming paper (Bensby, Feltzing, \& Oey, in prep.).

\section{References}

[1] D. Burstein, ApJ 234, 829 (1979)

[2] G. Gilmore, N. Reid, MNRAS 202, 1025 (1983)

[3] C. Soubiran, O. Bienaymé, A. Siebert, A\&A 398, 141 (2003),

[4] K. Fuhrmann, A\&A 338, 161 (1998)

[5] T. Bensby, S. Feltzing, I. Lundström, A\&A 421, 969 (2004)

[6] G. Gilmore, R.F.G. Wyse, J.B. Jones, AJ 109, 1095 (1995)

[7] B.E. Reddy, D.L. Lambert, C. Allende Prieto, MNRAS 367, 1329 (2006)

[8] T. Bensby, S. Feltzing, I. Lundström, A\&A 410, 527 (2003)

[9] T. Bensby, S. Feltzing, I. Lundström, I. Ilyin, A\&A 433, 185 (2005)

[10] T. Bensby, A.R. Zenn, M.S. Oey, S. Feltzing, ApJ 663, L13 (2007)

[11] T. Bensby, D. Adén, J. Meléndez, A. Gould, S. Feltzing, M. Asplund, J.A. Johnson, S. Lucatello, J.C. Yee, I. Ramírez et al., A\&A 533, A134 (2011)

[12] V. Hill, A. Lecureur, A. Gomez, M. Zoccali, M. Schultheis, C. Babusiaux, F. Royer, B. Barbuy, F. Arenou, D. Minniti et al., arXiv:1107.5199 (2011)

[13] T. Bensby, S. Feltzing, J.A. Johnson, A. Gould, D. Adén, A. M., J. Meléndez, A. Gal-Yam, S. Lucatello, H. Sana et al., A\&A 512, A41 (2010)

[14] A. Alves-Brito, J. Meléndez, M. Asplund, I. Ramírez, D. Yong, A\&A 513, A35 (2010)

[15] B. Nordström, M. Mayor, J. Andersen, J. Holmberg, F. Pont, B.R. Jørgensen, E.H. Olsen, S. Udry, N. Mowlavi, A\&A 418, 989 (2004)

[16] L. Casagrande, R. Schönrich, M. Asplund, S. Cassisi, I. Ramirez, J. Melendez, T. Bensby, S. Feltzing, A\&A 530, A138 (2011)

[17] T.V. Mishenina, C. Soubiran, V.V. Kovtyukh, S.A. Korotin, A\&A 418, 551 (2004)

[18] K. Fuhrmann, Astronomische Nachrichten 325, 3 (2004)

[19] B.E. Reddy, The Galactic Thick Disk: An Observational Perspective, in IAU Symposium, edited by K. Cunha, M. Spite, \& B. Barbuy (2010), Vol. 265 of IAU Symposium, pp. 289-299

[20] T. Bensby, M.S. Oey, S. Feltzing, B. Gustafsson, ApJ 655, L89 (2007)

[21] S. Feltzing, J. Holmberg, J.R. Hurley, A\&A 377, 911 (2001)

[22] B.E. Reddy, J. Tomkin, D.L. Lambert, C. Allende Prieto, MNRAS 340, 304 (2003)

[23] K. Fuhrmann, MNRAS 384, 173 (2008) 
[24] B. Edvardsson, J. Andersen, B. Gustafsson, D.L. Lambert, P.E. Nissen, J. Tomkin, A\&A 275, 101 (1993)

[25] T. Bensby, A. Alves-Brito, M.S. Oey, D. Yong, J. Meléndez, A\&A 516, L13 (2010)

[26] T. Bensby, A. Alves-Brito, M.S. Oey, D. Yong, J. Meléndez, ApJ 735, L46 (2011)

[27] P.J. Quinn, L. Hernquist, D.P. Fullagar, ApJ 403, 74 (1993)

[28] Á. Villalobos, S. Kazantzidis, A. Helmi, ApJ 718, 314 (2010)

[29] M.G. Abadi, J.F. Navarro, M. Steinmetz, V.R. Eke, ApJ 597, 21 (2003)

[30] F. Bournaud, B.G. Elmegreen, M. Martig, ApJ 707, L1 (2009), 0910.3677

[31] S.R. Loebman, R. Roškar, V.P. Debattista, Ž. Ivezić, T.R. Quinn, J. Wadsley, ApJ 737, 8 (2011) 